\title{
Evaluación de la actividad depresora del mentol en trucha arcoíris (Oncorhynchus mykiss) en diferentes condiciones de pH
}

\author{
Evaluation of menthol depressant activity in rainbow trout (Oncorhynchus mykiss) \\ under different $\mathrm{pH}$ conditions
}

\author{
Natalia Urzúa ${ }^{1,3}$, Miguel Mancinii ${ }^{1}$, Carlos Lüders $^{2}$, Carlos Errecalde', \\ Guillermo Prieto ${ }^{1}$
}

\section{Resumen}

Se evaluó la acción de mentol sobre variables fisiológicas y anestesiológicas en truchas arcoíris. Se trabajó con truchas juveniles clínicamente sanas, de ambos sexos, conformadas en grupo $\mathrm{A}(\mathrm{n}=20)$ de $201.4 \pm 60.1 \mathrm{~g}$ de peso sometidas a $100 \mathrm{ppm} / \mathrm{L}$ de mentol en agua a $\mathrm{pH} 6.4$ y en grupo $\mathrm{B}(\mathrm{n}=20)$ de $187.3 \pm 48.6 \mathrm{~g}$ de peso expuestos a idéntica concentración de mentol con el agua ajustada a pH 8.0 con bicarbonato de sodio. Los peces se ubicaron en recipientes con agua a $16.7^{\circ} \mathrm{C}$ y conductividad eléctrica de $0.10 \mathrm{mS} \mathrm{cm} \mathrm{cm}^{-1}$. La metodología de trabajo fue: 1) aclimatación de peces en un recipiente sin fármaco y registro de la frecuencia respiratoria basal (FR) tras adoptar un comportamiento normal de natación; 2) transferencia a un segundo recipiente con mentol y registro de tiempos de pérdida de equilibrio lateral y de anestesia; 3 ) traspaso de peces anestesiados al tercer recipiente sin fármaco y registro de FR y su comportamiento durante el periodo de recuperación. En el grupo A la anestesia se logró en $127.7 \pm 46.3 \mathrm{~s}$ con fuerte reducción de la FR basal de $132.4 \pm 12.6$ a $40.4 \pm 14.4 \mathrm{mov} / \mathrm{min}$ y la recuperación total se produjo en $189.7 \pm 64.2 \mathrm{~s}$. En el grupo B se observó tiempo de anestesia y

\footnotetext{
${ }^{1}$ Departamento de Clínica Animal, Facultad de Agronomía y Veterinaria, Universidad Nacional de Río Cuarto, Argentina

${ }^{2}$ Departamento de Ciencia Veterinaria, Facultad de Recursos Naturales, Universidad Católica de Temuco, Chile

${ }^{3}$ E-mail: nurzuapizarro@ayv.unrc.edu.ar
}

Recibido: 10 de enero de 2021

Aceptado para publicación: 15 de junio de 2021

Publicado: 24 de agosto de 2021

CLos autores. Este artículo es publicado por la Rev Inv Vet Perú de la Facultad de Medicina Veterinaria, Universidad Nacional Mayor de San Marcos. Este es un artículo de acceso abierto, distribuido bajo los términos de la licencia Creative Commons Atribución 4.0 Internacional (CC BY 4.0) [https:// creativecommons.org/licenses/by/4.0/deed.es] que permite el uso, distribución y reproducción en cualquier medio, siempre que la obra original sea debidamente citada de su fuente original 
recuperación a los $93.4 \pm 34.3$ y $181.7 \pm 68.0 \mathrm{~s}$, respectivamente, y reducción de la FR desde $137 \pm 14.3$ a $43.6 \pm 10.4 \mathrm{mov} / \mathrm{min}$. Los resultados indican que mentol en agua ajustado a pH 8.0 es una buena alternativa para las prácticas de piscicultura de truchas arcoíris.

Palabras clave: trucha arco iris, mentol, anestesia, $\mathrm{pH}$

\section{AbSTRACT}

The action of menthol on physiological and anesthesiological variables in rainbow trout was evaluated. Clinically healthy juvenile trout were used, of both sexes, formed in group $A(n=20)$ of $201.4 \pm 60.1 \mathrm{~g}$ of weight subjected to $100 \mathrm{ppm} / \mathrm{L}$ of menthol in water at pH 6.4 and in group $\mathrm{B}(\mathrm{n}=20)$ of $187.3 \pm 48.6 \mathrm{~g}$ of weight exposed to identical concentration of menthol with the water adjusted to $\mathrm{pH} 8.0$ with sodium bicarbonate. The fish were placed in containers with water at $16.7^{\circ} \mathrm{C}$ and electrical conductivity of $0.10 \mathrm{mScm}^{-1}$. The work methodology was: 1) acclimatization of fish in a container without drug and recording of the basal respiratory rate (RR) after adopting a normal swimming behavior; 2) transfer to a second container with menthol and recording of times of loss of lateral balance and anesthesia; 3) transfer of anesthetized fish to a third container without drug and record of $\mathrm{RR}$ and its behavior during the recovery period. In group A, anesthesia was achieved in $127.7 \pm 46.3 \mathrm{~s}$ with a strong reduction in baseline $R R$ from $132.4 \pm 12.6$ to $40.4 \pm 14.4 \mathrm{mov} / \mathrm{min}$ and total recovery occurred in $189.7 \pm 64.2 \mathrm{~s}$. In group B, anesthesia and recovery time were observed at $93.4 \pm 34.3$ and $181.7 \pm 68.0 \mathrm{~s}$, respectively, and a reduction in RR from $137 \pm 14.3$ to $43.6 \pm 10.4 \mathrm{mov} / \mathrm{min}$. The results indicate that menthol in water adjusted to $\mathrm{pH} 8.0$ is a good alternative for rainbow trout fish farming practices.

Key words: rainbow trout, menthol, anesthesia, $\mathrm{pH}$

\section{INTRODUCCIÓN}

El empleo de fármacos anestésicos en piscicultura facilita procedimientos propios de la actividad como inseminación artificial, inducción del desove, determinación del peso y medidas corporales, biopsias, transporte y técnicas reproductivas, además de reducir la incidencia de traumas (Sneddon et al., 2016; Mazandarani y Hoseini, 2017). La elección del agente anestésico contempla parámetros como la eficacia, costos, disponibilidad, facilidad de uso y ausencia de efectos adversos sobre peces, humanos y medio ambiente (Ross y Ross 2008; Neiffer y Stamper, 2009; Msangi y Batka, 2015).
Recientemente, algunos aceites esenciales de origen vegetal han sido considerados como posibles anestésicos en peces debido su origen natural y beneficios tales como la acción antioxidante, la mitigación del estrés y los efectos inmunoreguladores (Zahl et al., 2012; Mazandarani y Hoseini, 2017; Freitas Souza et al., 2019; Hoseini et al., 2019).

El mentol es un alcohol terpeno monocíclico mayoritario en el aceite esencial de Mentha spp que posee propiedades antiespasmódicas, antiinflamatorias y anestésicas (Rozza et al., 2014; Mazandarani y Hoseini, 2017). Su actividad depresora es mediada por la modulación de receptores de tipo ácido $\gamma$-aminobutírico A(GABAA) (Watt 
et al., 2008; Kasai et al., 2014), mientras las propiedades analgésicas son originadas por la activación selectiva de receptores opioides ê (Galeotti et al., 2002) y por bloqueo de canales de sodio (Haeseler et al., 2002).

El mentol es seguro en humanos (Façanha y Gomes, 2005; Yadegarinia et al., 2006), se consume como saborizante de alimentos, es ingrediente en productos farmacéuticos antiinflamatorios de venta libre y en artículos de higiene bucal (Teta y Kaiser, 2019). El metabolismo rápido del mentol y la excreción completa dentro de las 48 horas posteriores a la exposición de los peces (Botrel et al., 2017), lo posicionan como un anestésico con ventajas en acuicultura (Barbosa et al., 2017). Su uso como anestésico ha sido evaluado en invertebrados marinos (Simões y Gomes, 2009) y en algunas especies de peces (Kasai et al., 2014; Mazandarani y Hoseini, 2017; Martins et al., 2018; Hoseini et al., 2019; Teta y Kaiser, 2019). Los ensayos realizados demostraron sus propiedades anestésicas, aunque revelan tiempos de inducción y de recuperación dispares que oscilan entre 1 y 6 minutos y entre 0.95 y 16.6 minutos, respectivamente, según la dosis utilizada y la temperatura del agua (Mazandarani y Hoseini, 2017; Martins et al., 2018; Hoseini et al. 2019).

En ejemplares juveniles de truchas arcoíris (Oncorhynchus mykiss) se demostraron efectos anestésicos en baños de inmersión con 80 ppm de mentol (Teta y Kaiser, 2019). Estos autores refieren tiempos de inducción y recuperación de $100 \pm 21 \mathrm{~s} \mathrm{y} \mathrm{de}$ $94 \pm 13 \mathrm{~s}$, respectivamente, y ausencia de efectos colaterales, aun en aplicaciones de $150 \mathrm{ppm}$ en truchas arcoíris. Existen variaciones sustanciales interespecíficas en respuesta a los agentes anestésicos (Harmon, 2009; Readman et al., 2017), además de grandes diferencias individuales dentro de cada especie; variaciones que pueden ser el resultado de las dosis aplicadas (Harmon, 2009) así como por diferencias farmacocinéticas o farmacodinámicas (Teta y Kaiser, 2019).
En los peces, estas diferencias pueden estar influenciadas por factores biológicos como edad, sexo, etapa de la vida, peso corporal, tasa de crecimiento, composición corporal, estado fisiológico y estado de salud, así como por factores ambientales como la temperatura del agua, salinidad, $\mathrm{pH}$ y nivel de oxígeno (Sneddon, 2012; Zahl et al., 2012). El objetivo de este estudio fue evaluar la efectividad anestésica y sedante del mentol y su seguridad en truchas arcoíris. Los escasos antecedentes del uso de mentol como depresor en truchas arcoíris motivaron esta experiencia con el propósito de evaluar su acción depresora por inmersión sobre ciertas variables fisiológicas y anestesiológicas en esta especie con y sin ajuste de $\mathrm{pH}$, considerando que el $\mathrm{pH}$ de las soluciones anestésicas de inmersión influye en su eficacia; específicamente, un descenso en el $\mathrm{pH}$ reduce la eficacia por mayor ionización (Neiffer y Stamper, 2009).

\section{Materiales y Métodos}

\section{Animales}

En el criadero de Boca del Río, ubicado en la provincia de Córdoba, Argentina, se seleccionaron al azar 40 truchas juveniles clínicamente sanas, de ambos sexos, de un estanque de cultivo de 1200 ejemplares de O. mykiss.

Con base en los datos de peso y talla de los peces, se calculó el índice de condición de Fulton (K) de los dos grupos experimentales. Este índice se utiliza para comparar la «condición» o «bienestar» de los peces (Cifuentes et al., 2012; Ndiaye et al., 2015). Su cálculo se realiza a partir de la ecuación $\mathrm{K}=100 * \mathrm{~W} / \mathrm{L}^{3}$, donde: $\mathrm{W}=$ Peso total en gramos y $\mathrm{L}=$ longitud total en centímetros.

\section{Agentes Anestésicos y Equipos}

Se preparó una solución madre de mentol $(250 \mathrm{mg} / \mathrm{l})$ disolviendo los cristales de mentol en etanol absoluto (Biopack ${ }^{\circledR}$, Argen- 
tina), esencial para facilitar su emulsificación y solubilidad en el agua (Teta y Kaiser, 2019). Las concentraciones finales de las soluciones de exposición se prepararon incorporando un volumen apropiado de solución madre de mentol en $30 \mathrm{~L}$ de agua, mezclando hasta obtener una emulsión homogénea.

Se utilizó una pesa de escala electrónica de sensibilidad de $0.001 \mathrm{~g}$ (Ohaus Explorer $($ ) y un ictiómetro milimétrico graduado para obtener el peso y la longitud de cada pez.

\section{Diseño Experimental}

La evaluación del depresor de mentol se llevó a cabo en recipientes plásticos de 50 $\mathrm{L}$ con $30 \mathrm{~L}$ de agua a $16.7^{\circ} \mathrm{C}$ y $0.10 \mathrm{mS} / \mathrm{cm}^{-}$ ${ }^{1}$ de conductividad eléctrica, dispuestos de tal manera que se lograra una rápida manipulación de los peces para reducir al mínimo el estrés y el tiempo de permanencia fuera del agua.

A fin de preservar la aclimatación de los peces, se utilizó el agua del canal que abastece a los estanques de cultivo. El agua del recipiente con la preparación anestésica se renovó completamente después del paso de tres peces y los dos recipientes auxiliares para el registro de datos se mantuvieron con una recirculación continua de agua.

Los peces fueron distribuidos en un grupo $\mathrm{A}(\mathrm{n}=20)$ sometidos a $100 \mathrm{ppm}$ de mentol en el agua a pH 6.4 y en un grupo $\mathrm{B}(\mathrm{n}=20)$ expuestos a la misma concentración de mentol y pH 8.0 ajustado con bicarbonato de sodio.

\section{Actividad Anestésica}

En un primer recipiente, solo con agua (etapa 1), se colocaron los peces, uno a la vez, hasta que se observó un comportamiento de natación normal para determinar la ausencia o presencia de signos de excitación y evaluar la frecuencia respiratoria basal. En un segundo recipiente con anestésico con o sin regulación del $\mathrm{pH}$ (etapa 2) se registraron las respuestas fisiológicas de acuerdo con los protocolos establecidos por otros autores (Stoskopf y Posner 2008; Mazandarani y Hoseini, 2017; Teta y Kaiser, 2019).

Se determinaron los tiempos de pérdida parcial de equilibrio, de pérdida de equilibrio lateral y el tiempo de inducción de la anestesia; considerando estado de anestesia cuando los peces perdieron el sentido del equilibrio, la capacidad de natación y no presentaron reflejos al recibir la presión táctil de un operador en el pedúnculo caudal (Sneddon, 2012). Una vez alcanzada la anestesia, los peces fueron retirados para su medición y pesaje, e inmediatamente transferidos a un tercer recipiente libre del anestésico (etapa 3), para registrar la frecuencia respiratoria de la anestesia y el tiempo de recuperación que corresponde al tiempo transcurrido desde la salida de los peces del baño de anestesia hasta su total recuperación, cuando alcanzan el equilibrio y la natación normal activa. Por último, los peces expuestos se colocaron en jaulas flotantes etiquetadas y se les controló diariamente para determinar la mortalidad y los cambios en el comportamiento de alimentación durante un periodo de 48 horas.

\section{Análisis Estadístico}

Se realizó la prueba $\mathrm{T}$ para observar si los peces utilizados en ambos lotes son homogéneos en peso y longitud, mientras que, para los momentos de pérdida parcial de equilibrio, pérdida de equilibrio lateral, inducción de anestesia, recuperación, frecuencia respiratoria basal y en la anestesia se realizó un análisis no paramétrico utilizando la prueba de Mann-Whitney y la prueba U para determinar las diferencias significativas $(p<0.05)$ entre el grupo A y B. Todos los análisis se realizaron en el software SPSS 2.0. 

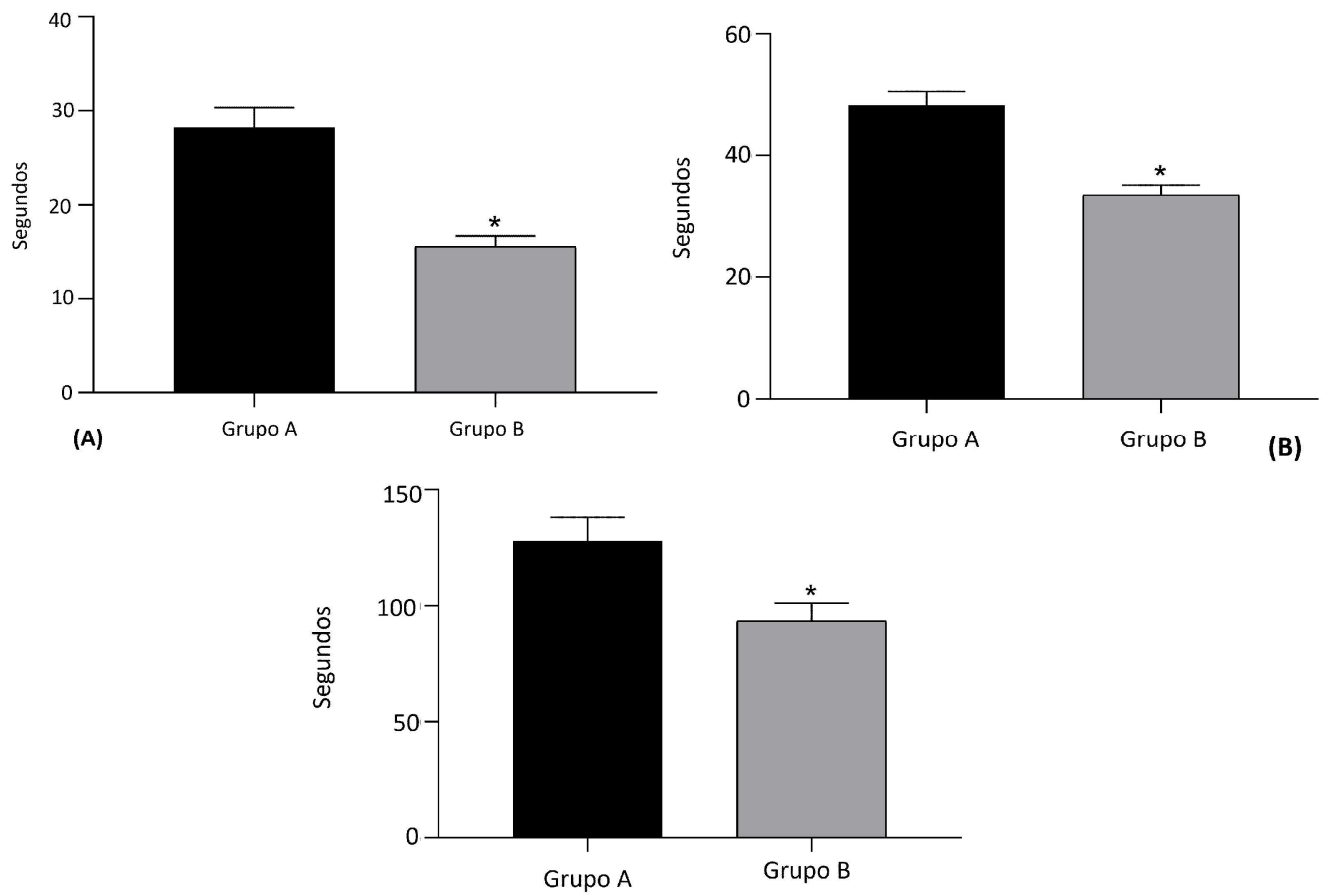

Figura 1. Tiempo pérdida de equilibrio parcial (A), tiempo de pérdida equilibrio lateral (B) y tiempo de anestesia $(\mathrm{C})$ en truchas de los grupos A y B (100 ppm de mentol disuelto en el agua con $\mathrm{pH} 6.4$ y 8 , respectivamente $(\mathrm{p}<0.05)(\mathrm{n}=20$ por grupo $)$

\section{Resultados}

No hubo diferencias significativas entre el grupo Ay B en relación con el peso y la longitud de los peces. El índice de Fulton (K) calculado para los grupos A y B dio proporciones de 1.43 y 1.42 , respectivamente.

La inducción anestésica rápida se presenta en ambos grupos; sin embargo, en el grupo $\mathrm{B}$ ( $\mathrm{pH} 8.0$ ), la pérdida parcial de equilibrio $(15.5 \pm 5.1 \mathrm{~s})$ (Figura 1A), la pérdida de equilibrio lateral ( $48.5 \pm 10.1 \mathrm{~s}$ ) (Figura 1B) y el tiempo de anestesia (127.7 $\pm 46.3 \mathrm{~s}$ ) (Figura $1 \mathrm{C})$ son significativamente inferiores $(\mathrm{p}<0.05)$ a los registrados en el grupo A (de $28.2 \pm 9.4 \mathrm{~s}, 33.5 \pm 7.1 \mathrm{~s}$ y $93.4 \pm 34.3 \mathrm{~s}$, respectivamente)

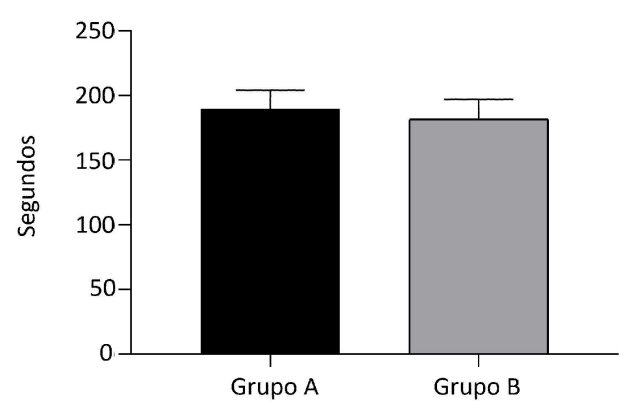

Figura 2. Tiempos de recuperación (RT), en truchas del grupo A y B (100 ppm de mentol disuelto en el agua con $\mathrm{pH} 6.4$ y 8 , respectivamente $(\mathrm{n}=20$ por grupo $)(\mathrm{p}>0.05)$

El tiempo de recuperación de la anestesia en los peces el grupo A fue de $189.7 \pm$ $64.2 \mathrm{~s}$ y grupo B $181.7 \pm 68.0 \mathrm{~s}$ (Figura 2), $\mathrm{y}$ las tasas respiratorias basal y de anestesia 


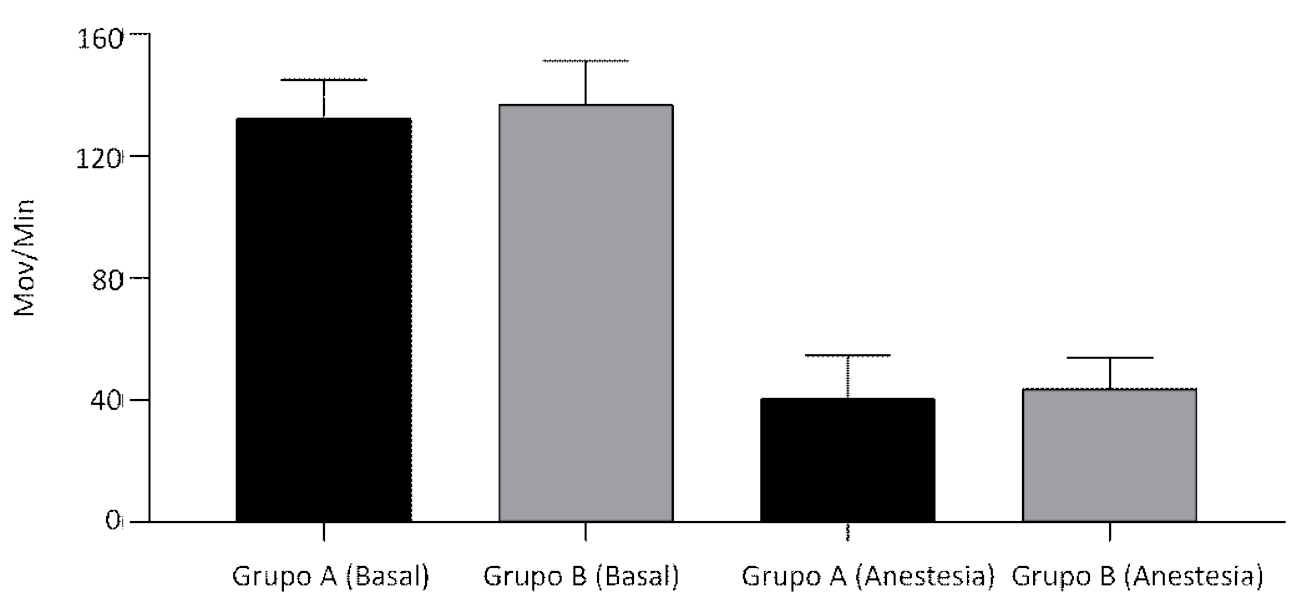

Figura 3. Frecuencia respiratoria (movimientos operculares por minuto) basal y durante la anestesia (An) en peces del grupo A y B (100 ppm de mentol disuelto en el agua con $\mathrm{pH} 6.4$ y 8 , respectivamente $(n=20$ por grupo) $(\mathrm{p}>0.05)$

(Figura 3) no registraron diferencias significativas $(\mathrm{p}>0.05)$. La frecuencia respiratoria basal y en anestesia del grupo A fue de 132.4 \pm 12.6 y $40.4 \pm 14.4 \mathrm{mov} / \mathrm{min}$, respectivamente y de los peces del grupo B fue de 137 \pm 14.3 y $43.6 \pm 10.4 \mathrm{mov} / \mathrm{min}$, respectivamente. Se observó una marcada disminución de la tasa respiratoria de la anestesia en ambos grupos, alcanzando valores de $69.4 \%$ y $68.1 \%$, para A y B, respectivamente. No se registraron muertes dentro de las 48 horas de terminada la experiencia.

\section{Discusión}

En cuanto al índice de Fulton, Cifuentes et al. (2012) y Ndiaye et al. (2015) proponen un valor de índice de e» 1 como valor de referencia del estado de bienestar. Con base a esto, los resultados obtenidos indican que las truchas estaban en buena condición corporal durante el estudio.

El uso de anestésicos de origen vegetal tiene un gran potencial en la acuicultura sobre los agentes sintéticos, que en algunos casos están prohibidos por problemas de seguridad y de residuos (Purbosari et al., 2019).
Se han estudiado varios extractos vegetales esenciales como anestésicos en peces; sin embargo, existen limitaciones en el caso de las plantas y especies estudiadas, habiéndose demostrado la eficacia en un pequeño número de especies piscícolas, pero, además, se desconocen los mecanismos de acción y las posibles vías metabólicas, necesitándose más datos para apoyar la seguridad y los efectos beneficiosos de estos anestésicos. Solo el metansulfonato de tricaína ha sido certificado por la FDA y la Unión Europea para su uso en el pescado destinado al consumo humano (Mazandarani y Hoseini, 2017).

El mentol a $100 \mathrm{ppm}$ de agua ajustada a $\mathrm{pH} 8.0$ constituye una buena alternativa para las prácticas de cultivo de trucha arcoíris, mejorando significativamente la inducción a la anestesia $(\mathrm{p}<0.05)$, sin modificar la frecuencia respiratoria, en comparación con el lote sin ajuste de $\mathrm{pH}$. Esto posiblemente como consecuencia del medio alcalino que reduce la ionización del fármaco, facilitando el paso del anestésico a través de las branquias (y probablemente la piel) y su difusión al sistema nervioso (Neiffer y Stamper, 2009; Sneddon, 2012; Zahl et al., 2012). 
El mentol es seguro, lo que se confirma por la ausencia de muertes, tanto en este estudio como en otros realizados en peces (Purbosari et al., 2019; Teta y Kaiser 2019). Los registros obtenidos son prometedores en comparación con los notificados en otras especies de peces (Mazandarani y Hoseini, 2017; Martins et al., 2018; Hoseini et al., 2019), y son similares a los reportados en $O$. mykiss por Teta y Kaiser (2019). Las diferencias observadas podrían atribuirse a las dosis de mentol utilizadas (Harmon, 2009); no obstante, coinciden con los criterios propuestos para que un anestésico pueda ser utilizado en acuicultura en cuanto a su efectividad, descrita como la capacidad de producir un estado de anestesia en un periodo de tres minutos y que la recuperación de la natación no exceda de 10 minutos (Ross y Ross, 2008; Neiffer y Stamper, 2009; Msangi y Batka, 2015).

\section{ConClusiones}

- El mentol tiene potencial como anestésico eficaz para la realización de tareas relacionadas al manejo de truchas de cultivo y puede considerárselo como una droga segura en la especie, y de fácil preparación en condiciones de campo.

- El mentol se mostró como un agente eficaz para alcanzar la anestesia, independiente del $\mathrm{pH}$, pero con mejores resultados a un $\mathrm{pH} 8$.

\section{Literatura Citada}

1. Barbosa LA, Maltez, LC, Stringhetta GR, Garcia L, Monserrat JM, da Silva DT, Heinzmann BM, et al. 2017. Properties of two plant extractives as anaesthetics and antioxidants for juvenile tambaqui Colossoma macropomum. Aquaculture 469: 79-87. doi: 10.1016/ j.aquaculture.2016.12.012
2. Botrel BM, Abreu DC, Saczk AA, Bazana MJ, Coelho SM, E Rosa PV, Magriotis ZM, et al. 2017. Residual determination of anesthetic menthol in fishes by SDME/GC-MS. Food Chem 229: 674-679. doi: 10.1016/j.foodchem.2017.02.087

3. Cifuentes R, González J, Montoya G, Jara A, Ortíz N, Piedra P, Habi TE. 2012. Relación longitud-peso y factor de condición de los peces nativos del río San Pedro (cuenca del río Valdivia, Chile). Gayana 76: 86-100. doi: 10.4067/S071765382012000100009

4. Façanha MF, Gomes LC. 2005. A eficácia do mentol como anestésico para tambaqui (Colossoma macropomum: Characiformes: Characidae). Acta Amazon 35: 71-75.

5. Galeotti N, Di Cesare Mannelli L, Mazzanti G, Bartolini A, Ghelardini $C$. 2002. Menthol: a natural analgesic compound. Neurosci Lett 322: 145-148. doi: 10.1016/s0304-3940(01)02527-7

6. Haeseler G, Maue D, Grosskreutz, J, Bufler J, Nentwig B, Piepenbrock S, Dengler R, Leuwer M. 2002. Voltagedependent block of neuronal and skeletal muscle sodium channels by thymol and menthol. Eur J Anaesthesiol 19: 571-579. doi: 10.1017/s02650215020009237.

7. Harmon TS. 2009. Methods for reducing stressors and maintaining water quality associated with live fish transport in tanks: a review of the basics. Rev Aquacult 1: 58-66. doi: 10.1016/j.aquaculture.2016.12.012

8. Hoseini S, Taheri A, Yousefi M. 2019. Application of herbal anaesthetics in aquaculture. Rev Aquacult 11: 550-564. doi: 10.1111/raq.12245

9. Kasai M, Hososhima S, Yun-Fei L. 2014. Menthol induces surgical anesthesia and rapid movement in ûshes. Open Neurosci J 8: 1-8. doi: 10.2174/ 1874082001408010001

10. Martins T, Valentim A, Pereira N, Antunes LM. 2019. Anaesthetics and analgesics used in adult fish for research: 
a review. Lab Anim 53: 325-341. doi: 10.1177/0023677218815199

11. Mazandarani M, Hoseini SM. 2017. Menthol and 1,8-cineole as new anaesthetics in common carp, Cyprinus carpio (Linnaeus, 1758). Aquac Res 48: 3041-3051. doi: 10.1111/are.13136

12. Msangi S, Batka M. 2015. The rise of aquaculture: the role of ûsh in global food security. In: 2014-2015 Global food policy report. [Internet]. Available in: https:// www.ifpri.org/publication/2014-2015global-food-policy-report

13. Ndiaye W, Diouf K, Samba O, Ndiaye P, Panfil J. 2015. The length-weight relationship and condition factor of white grouper (Epinephelus aeneus, Geoffroy Saint Hilaire, 1817) at the south-west coast of Senegal, West Africa Int J Adv Res (Indore) 3: 145-153.

14. Neiffer D, Stamper MA. 2009. Fish sedation, anesthesia, analgesia, and euthanasia: considerations, methods, and types of drugs. ILAR J 50: 343-360. doi: 10.1093/ilar.50.4.343

15. Purbosari N, Warsikic E, Syamsuc K, Santos $J$. 2019. Natural versus synthetic anesthetic for transport of live ûsh: a review. Aquac Fish 4: 129-133. doi: 10.1016/j.aaf.2019.03.002

16. Readman G, Owen S, Knowles T, Murrel JC. 2017. Species specific anaesthetics for fish anaesthesia and euthanasia. Sci Rep-UK 7: 7102. doi: 10.1038/s41598-017-06917-2

17. Ross L, Ross B. 2008. Anaesthetic and sedative techniques for aquatic animals. $3^{\text {rd }}$ ed. Oxford, UK: Blackwell. Publishing Ltd. 222 p.

18. Rozza AL, Meira de Faria F, Souza Brito AR, Pellizzon CH. 2014. The gastroprotective effect of menthol: involvement of antiapoptotic, antioxidant and anti-inflammatory activities. Plos One. 9: e86686. doi: 10.1371/journal.-pone.008-6686

19. Simões LN, Gomes LC. 2009. Eficácia do mentol como anestésico para juvenis de tilápia-do-nilo (Oreochromis niloti- cus). Arq Bras Med Vet Zoo 61: 613620. doi: 10.1590/S0102-09352009000300014

20. Sneddon LU. 2012. Clinical anesthesia and analgesia in fish. J Exot Pet Med 21: 32-43. doi: 10.1053/j.jepm.2011.11.009

21. Sneddon L, Wolfenden D, Thomson J. 2016. Stress management and welfare. In Fish physiology: biology of stress in fish. Cambridge: Academic Press. p 463539.

22. Souza CF, Baldissera MD, Baldisserotto B, Heinzmann BM, MartosSitcha JA, Mancera JM. 2019. Essential oils as stress-reducing agents for fish aquaculture: a review. Front Physiol 10: 785. doi: 10.3389/ fphys.2019.00785

23. Stoskopf M, Posner L. 2008. Anesthesia and restraint of laboratory ûsh. In: anesthesia and analgesia in laboratory animal. London, UK: Academic Press. p 519-534.

24. Teta C, Kaiser K. 2019. Menthol as an alternative anaesthetic and sedative for rainbow trout, Oncorhynchus mykiss. Afr J Aquat Sci 44: 71-76. doi: 10.2989/ 16085914.2018.1548342

25. Watt EE, Betts BA, Kotey FO, Humbert DJ, Griffith TN, Kelly EW, Veneskey KC, et al. 2008. Menthol shares general anesthetic activity and sites of action on the GABA(A) receptor with the intravenous agent, propofol. Eur J Pharmacol 590: 120-126. doi: 10.1016/j.ejphar.2008.06.003

26. Yadegarinia D, Gachkar L, Rezaei MB, Taghizadeh M, Astaneh SA, Rasooli I. 2006. Biochemical activities of Iranian Mentha piperita L. and Myrtus communis L. essential oils. Phytochemistry 67: 1249-1255. soi: 10.1016/j.phytochem.2006.04.025

27. Zahl IH, Samuelsen O, Kiessling A. 2012. Anaesthesia of farmed fish: implications for welfare. Fish Physiol Biochem38:201-218. doi: 10.1007/s10695011-9565-1 